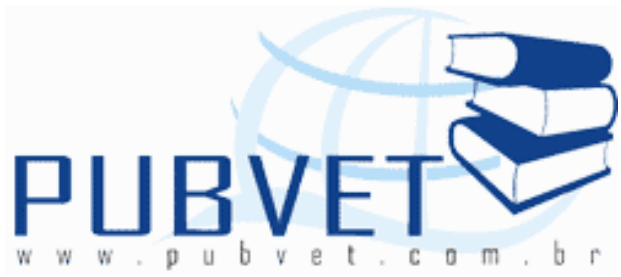

PUBVET, Publicações em Medicina Veterinária e Zootecnia.

\title{
Resposta de Pueraria phaseoloides (Benth.) à adubação fosfatada em presença ou não de fungo micorrízico
}

\author{
Newton de Lucena Costa ${ }^{1}$, Valdinei Tadeu Paulino ${ }^{2}$, Marcia Atauri Cardelli \\ Lucena $^{2}$, Roza Schunke ${ }^{3}$, Ruy Bessa Lopes ${ }^{4}$, João Avelar Magalhães ${ }^{5}$, Lucia \\ Elenícia da Silva Nascimento ${ }^{6}$
}

${ }^{1}$ Eng. Agr., M.Sc., Embrapa Roraima. Boa Vista, Roraima. Doutorando em Agronomia/Produção Vegetal, UFPR. Curitiba, Paraná.

${ }^{2}$ Eng. Agr., Ph.D., Instituto de Zootecnia. Nova Odessa, São Paulo.

${ }^{3}$ Eng. Agr., Ph.D., Embrapa Gado de Corte. Campo Grande, Mato Grosso do Sul.

${ }^{4}$ Biológo, D.Sc., Universidade Federal do Oeste do Pará. Santarém, Pará.

${ }^{5}$ Médico Veterinário, D.Sc., Embrapa Meio-Norte. Parnaíba, Piauí.

${ }^{6}$ Estudante de Pós-Graduação em Gestão Ambiental e Ecoturismo, Faculdade Montenegro. Parnaíba, Piauí. Laboratório de Água e Solos, Embrapa MeioNorte/UEP de Parnaíba. Parnaíba, Piauí.

\section{Resumo}

Os efeitos da inoculação do fungo micorrízico Gigaspora margarita e da aplicação de fosfato de rocha sobre o crescimento, nodulação, concentração e absorção de nitrogênio $(\mathrm{N})$ e fósforo $(\mathrm{P})$ em Pueraria phaseoloides cV. CIAT9900 foram avaliados em condições de casa de vegetação, utilizando-se um solo Latossolo Amarelo, textura argilosa e pobre em fósforo disponível (2 
COSTA, N.L. et al. Resposta de Pueraria phaseoloides (Benth.) à adubação fosfatada em presença ou não de fungo micorrízico. PUBVET, Londrina, V. 6, N. 9, Ed. 196, Art. 1315, 2012.

ppm). O delineamento experimental foi em blocos casualizados com cinco repetições. As plantas inoculadas com fungo micorrízico receberam $10 \mathrm{~g}$ de inóculo/vaso (raiz + solo + esporos). O fosfato de rocha foi aplicado na dosagem de $22 \mathrm{Kg}$ de P/ha. A micorrização, na presença de fosfato de rocha, resultou nos maiores $(P<0,05)$ rendimentos de matéria seca, teores de $P$, quantidades absorvidas de $\mathrm{P}$ e $\mathrm{N}$, número e peso de nódulos, enquanto que os teores de $\mathrm{N}$ não foram afetados $(P>0,05)$ pelos diferentes tratamentos.

Palavras-chave: fosfato de rocha, kudzu tropical, leguminosa

\section{Responses of Pueraria phaseoloides to phosphate fertilization with or without mycorrhizal inoculation}

\section{Abstract}

Responses of Pueraria phaseoloides cV. CIAT-9900 to inoculation with the mycorriza fungi Gigaspora margarita and with added phosphate rock were studied in P-deficient yellow-latosol ( $2 \mathrm{ppm}$ ). The trial was carried out on greenhouse. The pots were on randomized blocks with five replications per treatment. The mycorrhizal inoculations were $10 \mathrm{~g} /$ pot consisted of spores, soil and infected root fragments. The phosphate rock was $22 \mathrm{~kg} \mathrm{P} / \mathrm{ha}$. The mycorrhized plants with added of rock phosphate were increased significantly $(\mathrm{P}<0.05)$ the growth (dry weight), $\mathrm{P}$ content uptake and nodulation, while $\mathrm{N}$ content there were no significant differences $(P>0.05)$ among the treatments. Keywords legume, rock phosphate, tropical Kudzu

\section{Inrodução}

A utilização de leguminosas forrageiras associadas com gramíneas tropicais representa uma alternativa de melhorar a qualidade das pastagens, resultando em economia de fertilização nitrogenada. O fósforo é, freqüentemente, um fator limitante ao crescimento de muitas leguminosas tropicais (JONES; FREITAS, 1970; PAULINO et al., 1986, COSTA et al., 2007). 
COSTA, N.L. et al. Resposta de Pueraria phaseoloides (Benth.) à adubação fosfatada em presença ou não de fungo micorrízico. PUBVET, Londrina, V. 6, N. 9, Ed. 196, Art. 1315, 2012.

Para o estabelecimento e crescimento ótimos, aplicações de doses adequadas de fertilizante fosfatado são requeridas.

As micorrizas arbusculares são associações simbióticas mutualísticas existentes entre certos fungos do solo e raízes de plantas superiores (SCHUSSLER et al., 2001, citado por CARNEIRO et al., 2010). Um possível mecanismo alternativo para maximizar a eficiência do fertilizante fosfatado é via inoculação com micorriza arbuscular (OLSEN; HABTE, 1995; COSTA et al., 2007; CARNEIRO et al., 2011). Os efeitos da micorriza decorrem, principalmente, da elevação na absorção e conseqüente crescimento da planta (HAYMAN, 1975). De modo geral, segundo Silveira (1992), citado por Colozzi Filho e Nogueira (2007), a micorrização resulta na ação direta do fungo na absorção de nutrientes e, indireta na fixação biológica de nitrogênio, mineralização e/ou solubilização de nutrientes na micorrizosfera, além de atuar na proteção contra fungos patogênicos.

De outro lado, a Pueraria phaseoloides é uma leguminosa promissora para as condições do Trópico Úmido (COSTA, 1990), devido a sua adaptação aos solos ácidos e de baixa fertilidade, e à sua agressividade, que the confere capacidade competitiva em relação às plantas invasoras (SOUZA FILHO et al., 1999). Ademais, vem sendo utilizada na adubação orgânica e, devido ao seu valor nutritivo, na alimentação de pequenos e grandes ruminantes (COSTA et al., 1997; MAGALHÃES et al., 2001; MONTEIRO et al., 2009). Vale destacar que há escassas informações sobre micorrizas em leguminosas forrageiras tropicais.

Diante do exposto, o presente trabalho teve como objetivo avaliar os efeitos da inoculação do fungo micorrízico Gigaspora margarita e da aplicação de fosfato de rocha sobre o crescimento, concentração e absorção de nitrogênio, fósforo e nodulação de $P$. phaseoloides cv. CIAT-9900. 
COSTA, N.L. et al. Resposta de Pueraria phaseoloides (Benth.) à adubação fosfatada em presença ou não de fungo micorrízico. PUBVET, Londrina, V. 6, N. 9, Ed. 196, Art. 1315, 2012.

\section{Material e Métodos}

O experimento foi conduzido em casa de vegetação na Embrapa Rondônia, em Porto Velho, num solo Latossolo Amarelo, textura argilosa com as seguintes características químicas: $\mathrm{pH}\left(\mathrm{H}_{2} \mathrm{O}\right)=4,5 ; \mathrm{Al}^{3+}=2,9 \mathrm{cmol} / \mathrm{dm}^{3}$; $\mathrm{Ca}+\mathrm{Mg}=1,2 \mathrm{cmol} / \mathrm{dm}^{3} ; \mathrm{P}=2,0 \mathrm{ppm}$ e $\mathrm{K}=75 \mathrm{ppm}$. Utilizaram-se vasos contendo $2 \mathrm{Kg}$ de solo seco.

O delineamento experimental foi em blocos casualizados com cinco repetições. Os tratamentos avaliados foram: Testemunha (sem adubação), adição de micorriza arbuscular (MA), adição de fosfato de rocha (FR) e micorriza arbuscular + fosfato de rocha $(M A+F R)$. Oito sementes escarificadas de Pueraria phaseoloides cv. CIAT 9900 foram semeadas por vaso e, 15 dias após a emergência foi realizado o desbaste deixando-se 3 plantas por vaso. As plantas foram diariamente irrigadas com água destilada, mantendo a umidade do solo próxima a capacidade de campo.

No tratamento com inoculação as plantas receberam $10 \mathrm{~g}$ de inóculo por vaso (raiz + solo + esporos) do fungo Gigaspora margarita. No tratamento com fosfato de rocha foi empregada a dosagem de $22 \mathrm{Kg}$ de $\mathrm{P} / \mathrm{ha}$, sob a forma de fosfato natural de Araxá ( $28 \%$ de $\mathrm{P}_{2} \mathrm{O}_{5}$ total, $6 \%$ de $\mathrm{P}_{2} \mathrm{O}_{5}$ solúvel e $43 \%$ de $\mathrm{CaO})$. A porcentagem de raízes micorrizadas foi determinada segundo Giovannetti e Mosse (1980) após a coloração das raízes (PHILLIPS; HAYMAN, 1970). As avaliações estatísticas basearam-se nas análises de variância e as médias foram comparadas pelo teste de Duncan.

\section{Resultados e Discussão}

A produção de matéria seca (MS), os teores e quantidades absorvidas de nitrogênio, nodulação e as taxas de colonização radiculares estão apresentadas na Figura 1 e Tabela 1. 


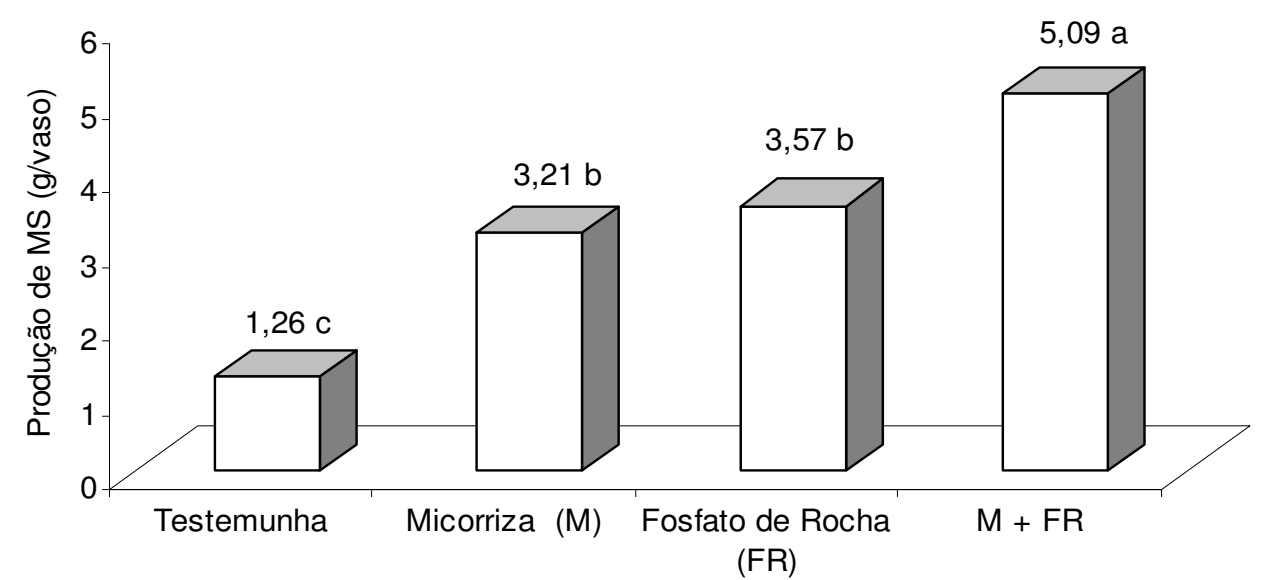

Figura 1. Produção de matéria seca (MS) da Pueraria phaseoloides cv. CIAT 9900, em função da micorrização e adubação fosfatada.

Isoladamente, tanto o tratamento com fosfato de rocha como a inoculação com micorrizas Gigaspora margarita, incrementaram a produção de MS, porém a combinação fosfato de rocha + micorriza arbuscular (FR + MA) resultou em maiores produções de MS e acumulações de nitrogênio. Não houve diferença significativa entre as médias dos tratamentos com micorriza ou com fosfato de rocha em relação à absorção de nitrogênio e nodulação (Tabelas 1 e 2). Resultados semelhantes foram relatados por Costa et al. (1992) avaliando o efeito de MA, na presença ou não de adubação fosfatada, em Leucaena leucocephala. Para Ázcon-Aguilar e Barea (1978), bactérias solubilizadoras de fosfatos estão presentes na rizosfera micorrízica atuando sinergisticamente com os endófitos. Deste modo, as MA ao aumentarem a absorção de fósforo, favorecem a dissociação química do fosfato insolúvel visando estabilizar sua concentração na solução do solo 
COSTA, N.L. et al. Resposta de Pueraria phaseoloides (Benth.) à adubação fosfatada em presença ou não de fungo micorrízico. PUBVET, Londrina, V. 6, N. 9, Ed. 196, Art. 1315, 2012.

Tabela 1. Teores e quantidades acumuladas de nitrogênio de Pueraria phaseoloides cv. CIAT 9900, em função da micorrização e adubação fosfatada.

\begin{tabular}{lcc}
\hline Tratamentos & Nitrogênio (\%) & $\mathrm{N}$ total (mg/vaso) \\
\hline Testemunha & $3,94^{\mathrm{a}}{ }^{\mathrm{a}}$ & $49,6^{\mathrm{c}}$ \\
Micorriza arbuscular (MA) & $117,5^{\mathrm{b}}$ \\
Fosfato de Rocha (FR) & $3,66^{\mathrm{a}}$ & $125,3^{\mathrm{b}}$ \\
MA + FR & $3,51^{\mathrm{a}}$ & $175,1^{\mathrm{a}}$ \\
\hline
\end{tabular}

- Médias seguidas pelas mesmas letras, nas colunas, não diferem entre si pelo teste de Duncan a $5 \%$ de probabilidade.

${ }^{1}$ Gigaspora margarita

A inoculação com micorrizas aumentou em 102,35\% o número de nódulos por planta (Tabela 2). Da mesma maneira o tratamento FR + M apresentou maior número de nódulos que a aplicação de cada tratamento isolado.

Tabela 2. Taxa de colonização radicular e nodulação da Pueraria phaseoloides cv. CIAT 9900, em função da micorrização e adubação fosfatada.

\begin{tabular}{|c|c|c|c|}
\hline \multirow[b]{2}{*}{ Tratamentos } & \multirow[b]{2}{*}{ Colonização } & \multicolumn{2}{|c|}{ Nodulação } \\
\hline & & Número & $\begin{array}{l}\text { Peso seco } \\
\text { (mg/vaso) }\end{array}$ \\
\hline Testemunha & - & $8,5^{\mathrm{c}}$ & $47^{d}$ \\
\hline Micorriza arbuscular (MA) ${ }^{1}$ & 56 & $17,2^{b}$ & $69^{c}$ \\
\hline Fosfato de Rocha (FR) & 31 & $26,4^{b}$ & $109^{b}$ \\
\hline $\mathrm{MA}+\mathrm{FR}$ & 49 & $41,2^{a}$ & $161^{\mathrm{a}}$ \\
\hline
\end{tabular}

A micorrização ou a adição de FR incrementaram significativamente, os teores de fósforo (P) na parte área da puerária (Tabela 3). Gerdemann e Trappe (1974) verificaram que as hifas do fungo que colonizavam o córtex estendem-se no solo adjascente, podendo atingir distâncias consideráveis (16 $\mathrm{cm}$ ) da superfície da raiz, aumentando, deste modo, a interface raiz-solo, além de fazerem a comunicação das raízes absorventes com zonas não esgotadas em nutrientes. 
COSTA, N.L. et al. Resposta de Pueraria phaseoloides (Benth.) à adubação fosfatada em presença ou não de fungo micorrízico. PUBVET, Londrina, V. 6, N. 9, Ed. 196, Art. 1315, 2012.

Tabela 3. Teores e quantidades absorvidas de fósforo da Pueraria phaseoloides cv. CIAT 9900, em função da micorrização e adubação fosfatada.

\begin{tabular}{lcc}
\hline Tratamentos & Fósforo (\%) & Fósforo absorvido (mg/vaso) \\
\hline Testemunha & $0,10^{\mathrm{c}}$ & $1,30^{\mathrm{c}}$ \\
Micorriza arbuscular (MA) & $0,14^{\mathrm{b}}$ & $4,33^{\mathrm{b}}$ \\
Fosfato de Rocha (FR) & $0,18^{\mathrm{a}}$ & $6,46^{\mathrm{b}}$ \\
MA + FR & $0,19^{\mathrm{a}}$ & $9,57^{\mathrm{a}}$ \\
\hline
\end{tabular}

- Médias seguidas pelas mesmas letras, nas colunas, não diferem entre si pelo teste de Duncan a $5 \%$ de probabilidade

${ }^{1}$ Gigaspora margarita

A colonização radicular pela MA melhorou a nutrição da puerária, principalmente em $\mathrm{P}$, que age imediatamente sobre o processo de fixação biológica, auxiliando nos requerimentos energéticos. Plantas não inoculadas com MA apresentaram crescimento reduzido e nodulação baixa, em ausência de fertilização fosfatada, devido a deficiência de $\mathrm{P}$ no solo. Esses resultados concordam com os obtidos por França e Carvalho (1970) e Paulino et al. (1986).

\section{Conclusões}

1 - A inoculação micorrízica influi positivamente sobre o crescimento e absorção de fósforo da puerária.

2 - As plantas micorrizadas acumulam mais nitrogênio e apresentaram maior nodulação.

\section{Referências Bibliográficas}

ÁZCON-AGUILAR, G.; BAREA, J.M. Effects of interaction between different culture fractions of phosphobacteria and Rhizobium on mycorrhizas infection growth and nodulation of Medicago sativa. Canadian Journal of Microbiology, v.24, p.520-524, 1978.

CARNEIRO, R.F.V.; MARTINS, M.A.; VÁSQUEZ, H.M.; DETMANN, E. Doses de fósforo e inoculação micorrízica no cultivo de estilosantes em solo sob condições naturais. Archivos de Zootecnia, v.59, n.227, p.415-426, 2010. 
CARNEIRO, R.F.V.; MARTINS, M.A.; ARAUJO, A.S.F.; NUNES, L.A.P. L. Inoculação micorrízica arbuscular e adubação fosfatada no cultivo de forrageiras consorciadas. Archivos de Zootecnia, v.60, n.232, p.1191-1202, 2011.

COLOZZI FILHO, A.; NOGUEIRA, M.A. Micorrizas arbusculares em plantas tropicais: café, mandioca e cana-de-açúcar. In: SILVEIRA, A.P.D.; FREITAS, S.S. (Org.). Microbiota do solo e qualidade ambiental. Campinas: Instituto Agronômico, 2007. 312p.

COSTA, N. de L. Pueraria: leguminosa forrageira para a produção de proteína. Porto Velho, EMBRAPA, UEPAE de Porto Velho, 1990. (Comunicado Técnico, 92).

COSTA, N. de L.; PAULINO, V.T.; VEASEY, E.A.; LEÔNIDAS, F. das C. Effect of vesiculararbuscular mycorrhiza and rock phosphate fertilization on growth, nodulation, and nitrogen and phosphorus uptake of leucaena. Leucaena Research Reports, v.13, p.10-12, 1992.

COSTA, N. de L.; TOWNSEND, C.R.; MAGALHÃES, J.A.; TAVARES, A.C.; PEREIRA, R.G.A. Utilização de bancos-de-proteína de Pueraria phaseoloides e Desmodium ovalifolium na alimentação de vacas leiteiras. In: CONGRESSO BRASILEIRO DE MEDICINA VETERINÁRIA, 25., 1997, Gramado. Anais... Gramado: SBMV, 1997. p. 264.

COSTA, N. L.; PAULinO, V. T.; TOWNSEND, C. R.; MAGALHAES, J. A. Resposta de Arachis pintoi cv. Amarillo a níveis de fósforo. Revista de Biologia e Ciências da Terra, v.6, n.1, p.59-62, 2006.

COSTA, N. de L.; MAGALHÃES, J.A.; PEREIRA, R.G.A.; TOWNSEND, C.R.; OLIVEIRA, J.R.C. Considerações sobre o manejo de pastagens na Amazônia Ocidental. Revista do Conselho Federal de Medicina Veterinária (CFMV), v.13, p.37-55, 2007.

FRANÇA, G.E.; CARVALHO, M.M. Ensaio exploratório de fertilização em cinco leguminosas tropicais em um solo de cerrado. Pesquisa Agropecuária Brasileira, v.5, p.147-153, 1970.

GERDEMANN, J.W.; TRAPPE, J.M. The endogonaceae in the Pacific Northwest. Mycological Memories, v.5, n.1, p.1-76, 1974.

GIOVANNETTI, M.; MOSSE, B. An evaluation of techniques for measuring vesicular-arbuscular mycorrhizal infection in roots. New Phytologist, v.84, n.3, p.489-500, 1980.

HAYMAN, D.S. Phosphorus cycling by soil microorganisms and plant roots. In: Soil Microbiology a critical review. Ed. N. Walkes, p.67-97, 1975.

JONES, M.B.; FREITAS, L.M.M. Respostas de quatro leguminosas tropicais a fósforo, potássio e calcáreo num latossolo Vermelho Amarelo de campo cerrado. Pesquisa Agropecuária Brasileira, v.5, p.91-99, 1970.

MAGALHÃES, J.A.; COSTA, N. de L.; PEREIRA, R.G.A.; TOWNSEND, C.R. Desempenho produtivo e reações fisiológicas de ovinos deslanados mantidos sob seringal (Hevea brasiliensis). Revista Científica de Produção Animal, v.3, n.1, p.77-82, 2001.

MONTEIRO, E.M.M.; LOURENÇO JÚNIOR, J.B.; SANTOS, N.F.A. dos; ARAÚJO, C.V.; FATURI, C.; GARCIA, A.R. Pueraria phaseoloides (Roxb.) Benth: alternativa na suplementação de ruminantes em períodos de escassez de forragem na Amazônia. Amazônia: Ciência e Desenvolvimento, v.4, n.8, p.217-228, 2009. 
PAULINO, V.T.; PICCINI, D.F.; BAREA, J.M. Influência de fungos micorrízicos vesículoarbusculares e fosfatos em leguminosas forrageiras tropicais. Revista Brasileira de Ciência do Solo, v.10, n.2, p.103-108, 1986.

PHILLIPS, J. M.; HAYMAN, D.S. Improved procedure for clearing roots and staining parasitic and vesicular-arbuscular mycorrhizal fungi for rapid assesment for infection. Transactions of the British Mycological Society, 55, n.1, p.158-161, 1970.

SOUZA FILHO, A.P. da; SILVA, M.A.M.; DUTRA, S. Quebra de dormência em sementes de Pueraria phaseoloides (Roxburgh) Bentham. Pasturas Tropicales, v.21, n.2, p.29-33, 1999. 